\title{
Article \\ Enhancing Ant-Based Algorithms for Medical Image Edge Detection by Admissible Perturbations of Demicontractive Mappings
}

\author{
Vasile Berinde ${ }^{1,2, *,+}+\mathbb{D}$ and Cristina Ţicală ${ }^{1,+} \mathbb{D}$ \\ 1 Department of Mathematics and Computer Science, North University Center at Baia Mare, \\ Technical University of Cluj-Napoca, Victoriei 76, 430072 Baia Mare, Romania; cristina.ticala@mi.utcluj.ro \\ 2 Academy of Romanian Scientists, Ilfov Str. no. 3, 050045 Bucharest, Romania \\ * Correspondence: vasile.berinde@mi.utcluj.ro or vberinde@cunbm.utcluj.ro \\ + These authors contributed equally to this work.
}

check for updates

Citation: Berinde, V.; Ţicală, C. Enhancing Ant-Based Algorithms for Medical Image Edge Detection by Admissible Perturbations of Demicontractive Mappings. Symmetry 2021, 13, 885. https:// doi.org/10.3390/sym13050885

Academic Editors: Wei-Shih Du; Alicia Cordero Barbero; Huaping Huang and Juan Ramón Torregrosa Sánchez

Received: 15 April 2021

Accepted: 12 May 2021

Published: 17 May 2021

Publisher's Note: MDPI stays neutral with regard to jurisdictional claims in published maps and institutional affiliations.

Copyright: (c) 2021 by the authors. Licensee MDPI, Basel, Switzerland. This article is an open access article distributed under the terms and conditions of the Creative Commons Attribution (CC BY) license (https:/ / creativecommons.org/licenses/by/ $4.0 /)$.

\begin{abstract}
The aim of this paper is to show analytically and empirically how ant-based algorithms for medical image edge detection can be enhanced by using an admissible perturbation of demicontractive operators. We thus complement the results reported in a recent paper by the second author and her collaborators, where they used admissible perturbations of demicontractive mappings as test functions. To illustrate this fact, we first consider some typical properties of demicontractive mappings and of their admissible perturbations and then present some appropriate numerical tests to illustrate the improvement brought by the admissible perturbations of demicontractive mappings when they are taken as test functions in ant-based algorithms for medical image edge detection. The edge detection process reported in our study considers both symmetric (Head CT and Brain CT) and asymmetric (Hand X-ray) medical images. The performance of the algorithm was tested visually with various images and empirically with evaluation of parameters.
\end{abstract}

Keywords: enriched demicontractive operator; edge detection; admissible perturbation; ant-based algorithm; test function; symmetric medical image; asymmetric medical image

\section{Introduction}

Iterative algorithms and, in particular, fixed point iterative algorithms, are important tools in the application of mathematical methods to problems arising in acoustic signal processing, optical imaging and medical tomography. Many of the problems and algorithms in the mathematics of medical image reconstruction are modelled by nonexpansive operators or nonexpansive type operators such that the resulting fixed point equations are in most cases solved by appropriate fixed point iterative algorithms (see, e.g., the monograph [1]).

In a recent paper [2], to enhance the quality of the edge detection results in medical image processing when using ant-based algorithms, the authors used as test functions admissible perturbations of some demicontractive operators.

The class of demicontractive mappings, which includes, among others, the class of nonexpansive mappings having a fixed point and also the class of quasi-nonexpansive mappings, turned out to provide very convenient attenuation properties for medical images edge detection when using ant-based algorithms, as illustrated by the numerical tests reported by [2] and in Section 3 of the present paper.

On the other hand, demicontractive mappings have been successfully used to solve nonlinear functional equations [3], fixed point problems [4-6], multiple-set split feasibility problems [7], equilibrium problems and variational inequality problems [8-10], as well as many other important linear and nonlinear problems from applied mathematics.

Starting from the large spectrum of interests related to demicontractive mappings, the aim of this research is twofold: first, to highlight some interesting properties of these 
mappings and, secondly, to illustrate-in conjunction with an appropriate admissible perturbation - their attenuation properties when used as test functions for ant-based algorithms in medical images edge detection. The edge detection process reported in our study considers both symmetric (Head CT and Brain CT) and asymmetric (Hand X-ray) medical images.

Our approach here is complementing the results reported in [2], where the authors studied the influence of choosing the appropriate parameter values for the quality of the edge detection algorithm. In the present paper, we focus on the process of selecting the test function used in building the heuristic. This work clearly shows that, in ant algorithms for medical images edge detection, the use of demicontractive operators and especially of the admissible perturbations of demicontractive operators is beneficial for the quality of the extracted edges.

To this end, in the next section, we give a brief account of the definition, connections and main properties of demicontractive mappings, as well as the notion and properties of the so-called admissible perturbation of an operator.

\section{Enriching Nonlinear Mappings by Admissible Perturbations}

Let $H$ be a real Hilbert space with norm and inner product denoted as usually by $\|\cdot\|$ and $\langle\cdot, \cdot\rangle$, respectively. Let $C \subset H$ be a closed and convex set and $T: C \rightarrow C$ be a self mapping and denote by

$$
\operatorname{Fix}(T)=\{x \in C: T x=x\}
$$

the set of fixed points of $T$. The mapping $T$ is called:

(1) nonexpansive if $\|T x-T y\| \leq\|x-y\|$, for all $x, y \in C$;

(2) quasi nonexpansive if Fix $(T) \neq \varnothing$ and $\|T x-y\| \leq\|x-y\|$, for all $x \in C$ and $y \in \operatorname{Fix}(T)$

(3) demicontractive $[4,6]$ if Fix $(T) \neq \varnothing$ and there exists $k<1$ such that

$$
\|T x-y\|^{2} \leq\|x-y\|^{2}+k\|x-T x\|^{2},
$$

for all $x \in C$ and $y \in \operatorname{Fix}(T)$, and we say $T$ is $k$-demicontractive;

(4) $k$-strictly pseudocontractive of the Browder-Petryshyn type [11] if there exists $k<1$ such that

$$
\|T x-T y\|^{2} \leq\|x-y\|^{2}+k\|x-y-T x+T y\|^{2}, \forall x, y \in C ;
$$

(5) hemicontractive if (1) holds with $k=1$; and

(6) pseudocontractive if (2) holds with $k=1$.

It is easily seen that any nonexpansive mapping with $\operatorname{Fix}(T) \neq \varnothing$ is quasi nonexpansive and that any quasi nonexpansive mapping is demicontractive, but the reverses may not be true, as illustrated by Example 1.

In addition, any nonexpansive mapping is $k$-strictly pseudocontractive of the BrowderPetryshyn type and hence pseudocontractive, but the reverses are not generally valid. Moreover, if we take $y \in$ Fix $(T)$ in (2), we see that any $k$-strictly pseudocontractive mapping of the Browder-Petryshyn type is $k$-demicontractive, but the reverse is no longer true.

Example 1. Let $H$ be the real line and $C=[0,1]$. Define $T$ on $C$ by $T x=\frac{2}{3} x \sin \frac{1}{x}$, if $x \neq 0$ and $T 0=0$. Then, Fix $(T)=\{0\}, T$ is demicontractive (and also quasi nonexpansive) but $T$ is not nonexpansive or pseudocontractive. Indeed, for $x \in C$ and $y=0$,

$$
|T x-0|^{2}=|T x|^{2}=\left|\frac{2}{3} x \sin (1 / x)\right|^{2} \leq\left|\frac{2}{3} x\right|^{2} \leq|x|^{2} \leq|x-0|^{2}+k|T x-x|^{2},
$$


for any $k<1$. Hence, (1) is satisfied. To see that $T$ is not nonexpansive, just take $x=\frac{2}{\pi}$ and $y=\frac{2}{3 \pi}$ to get

$$
|T x-T y|=\frac{16}{9 \pi}>\frac{4}{3 \pi}=|x-y| .
$$

With the same values for $x$ and $y$, we have

$$
|x-y|^{2}+|x-T x-y+T y|^{2}=\frac{160}{81 \pi^{2}}<\frac{256}{81 \pi^{2}}=|T x-T y|^{2},
$$

which shows that $T$ is not pseudocontractive.

Example 2. Let $H$ be the real line with the usual metric and define $S$ on $C=[0,1]$ by $S x=x^{2}$, $x \in C$. We have Fix $(S)=\{0,1\} \neq \varnothing$, but $T$ is not demicontractive.

Let now $(X, d)$ be a metric space. A mapping $T: X \rightarrow X$ is said to be asymptotically regular if, for each $x$ in $X$,

$$
d\left(T^{n+1} x, T^{n} x\right) \rightarrow 0 \text { as } n \rightarrow \infty .
$$

It is well known that any Banach contraction, i.e., any mapping $T: X \rightarrow X$, satisfying the Banach contraction condition

$$
d(T x, T y) \leq c d(x, y), x, y \in X \quad(0<c<1),
$$

is asymptotically regular.

Despite the fact that a nonexpansive mapping $T$ is the limit case $(c=1)$ of a Banach contraction mapping - which is asymptotically regular-a nonexpansive map is, in general, not asymptotically regular. This is fact is illustrated by the following two simple examples.

Example 3. (1) Let $X=\mathbb{R}$ with the usual norm and $T: X \rightarrow X$ be given by $T x=x+1, x \in X$. Then, $T^{n} x=x+n, n \geq 1$ and so $T^{n+1} x-T^{n} x=1$, for all $n \geq 1$ and hence $T$ is not asymptotically regular on $X$. Note that in this case Fix $(T)=\{x \in X: T x=x\}=\varnothing$.

(2) Let $X=\mathbb{R}$ with the usual norm and $T: X \rightarrow X$ be given by $T x=1-x, x \in X$. In this case, we have $T^{2 n+1} x=1-x, T^{2 n} x=x, n \geq 1$ and hence $T$ is not asymptotically regular on $X$, although Fix $(T)=\{1 / 2\}$.

Krasnosel'skiĩ [12] noted the fact that, for a nonexpansive mapping $T$, the averaged mapping associated to $T$, that is,

$$
T_{\lambda}:=(1-\lambda) I+\lambda T, \lambda \in(0,1),
$$

is asymptotically regular. Thus, by observing that

$$
\operatorname{Fix}(T)=\operatorname{Fix}\left(T_{\lambda}\right),
$$

for any $\lambda \in(0,1)$, in order to approximate the fixed points of $T$, one can use an enriched mapping, that is, the average mapping $T_{\lambda}$, which is asymptotically regular, while $T$ is not.

In this way, Krasnosel'skir [12] proved that the iterative process

$$
x_{n+1}=\frac{1}{2}\left(x_{n}+T x_{n}\right), n \geq 0,
$$

converges to a fixed point of a nonexpansive mapping $T$ (see also [11] for other related results).

On the other hand, Rus [13] introduced the concept of admissible perturbation of an operator, as an abstract approach to the study of fixed point iterative schemes. 
Definition 1. [13] Let $X$ be a nonempty set. A mapping $G: X \times X \rightarrow X$ is called admissible if it satisfies the following two conditions:

$\left(A_{1}\right) G(x, x)=x$, for all $x \in X$;

$\left(A_{2}\right) G(x, y)=x$ implies $y=x$.

Definition 2. [13] Let $X$ be a nonempty set. If $T: X \rightarrow X$ is a given operator and $G: X \times X \rightarrow X$ is an admissible mapping, then the operator $T_{G}: X \rightarrow X$, defined by

$$
T_{G}(x)=G(x, f(x)), \forall x \in X,
$$

is called the admissible perturbation of $T$.

Example 4. [13] Let $(V,+, \mathbb{R})$ be a real vector space, $X \subset V$ a convex subset, $\lambda \in(0,1)$, $T: X \rightarrow X$ and $G: X \times X \rightarrow X$ be defined by

$$
G(x, y):=(1-\lambda) x+\lambda y, x, y \in X
$$

Then, $T_{G}$ is an admissible perturbation of $T$. We denote in the following $T_{G}$ by $T_{\lambda}$ and call it the Krasnoselskij perturbation of $T$, in view of the pioneering results obtained by Krasnoselskij [12].

Remark 1. The following property of an admissible perturbation

$$
\operatorname{Fix}\left(T_{G}\right)=\operatorname{Fix}(T),
$$

expressed by (4) in the case of Krasnoselskij perturbation, is fundamental in the iterative approximation of fixed points.

Note that, in general,

$$
\operatorname{Fix}\left(T_{G}^{n}\right) \neq \operatorname{Fix}\left(T^{n}\right), n \geq 2 \text {. }
$$

Some other authors have studied various properties of the admissible perturbation of certain classes of operators: nonexpansive operators [14], $\phi$-pseudocontractive operators [15], nonself generalized pseudocontractive operators [16], $\alpha$ - $\psi$-pseudocontractive operators $[17,18]$, demicontractive operators [19,20], multivalued operators [21], etc.

From this point of view, some of the following properties of demicontractive mappings, on the one hand, and of admissible perturbations of a nonlinear operator, on the other hand, are important in our numerical applications reported in Section 3.

Proposition 1. Let $H$ be a real Hilbert space and $C \subset H$ be a closed and convex set. If $T: C \rightarrow C$ is a quasi-nonexpansive mapping, then, for any $\lambda \in(0,1)$, the Krasnoselskij perturbation $T_{\lambda}$ of $T$ is pseudocontractive.

Proof. By hypothesis, we have Fix $(T) \neq \varnothing$ and $\|T x-y\| \leq\|x-y\|$, for all $x \in C$ and $y \in \operatorname{Fix}(T)$, which is equivalent to

$$
\langle T x-x, x-y\rangle \geq 0, x \in C, y \in \operatorname{Fix}(T) .
$$

Then, for all $x \in C$ and $y \in$ Fix $(T)$, we have

$$
\begin{aligned}
\left\|T_{\lambda} x-y\right\|^{2}= & \|\lambda(T x-x)+x-y\|^{2}=\|x-y\|^{2}+2 \lambda\langle T x-x, x-y\rangle \\
& +\lambda^{2}\|T x-x\|^{2} \leq\|x-y\|^{2}+\lambda^{2}\|T x-x\|^{2} \\
& +\lambda^{2}\|T x-x\|^{2}=\|x-y\|^{2}+\left\|T_{\lambda} x-x\right\|^{2}
\end{aligned}
$$

which shows that $T_{\lambda}$ is pseudocontractive. 
Proposition 2. Let $H$ be a real Hilbert space and $C \subset H$ be a closed and convex set. If $T: C \rightarrow C$ is $k$-demicontractive, then the Krasnoselskij perturbation $T_{\lambda}$ of $T$ is $(1+k / \lambda-1 / \lambda)$ demicontractive.

Proof. By hypothesis, we have Fix $(T) \neq \varnothing$ and there exists $k<1$ such that

$$
\|T x-y\|^{2} \leq\|x-y\|^{2}+k\|x-T x\|^{2}
$$

for all $x \in C$ and $y \in$ Fix $(T)$, which is equivalent to

$$
\langle T x-x, x-y\rangle \leq \frac{k-1}{2} \cdot\|x-T x\|^{2}, x \in C, y \in \operatorname{Fix}(T) .
$$

Then, similar to the proof of Lemma 1 , for all $x \in C$ and $y \in$ Fix $(T)$, we have

$$
\begin{gathered}
\left\|T_{\lambda} x-y\right\|^{2}=\|\lambda(T x-x)+x-y\|^{2}=\|x-y\|^{2}+2 \lambda\langle T x-x, x-y\rangle \\
+\lambda^{2}\|T x-x\|^{2} \leq\|x-y\|^{2}+\left(\lambda^{2}+\lambda k-\lambda\right)\|T x-x\|^{2} \\
=\|x-y\|^{2}+\frac{\lambda^{2}+\lambda k-\lambda}{\lambda^{2}} \cdot\left\|T_{\lambda} x-x\right\|^{2}, x \in C, y \in \operatorname{Fix}(T),
\end{gathered}
$$

which shows that $T_{\lambda}$ is $(1+k / \lambda-1 / \lambda)$-demicontractive.

Remark 2. It is important to note that the Krasnoselskij perturbation of a k-demicontractive map has a better demicontractiveness constant, since

$$
1+\frac{k}{\lambda}-\frac{1}{\lambda}>k
$$

for any $k<1$.

Therefore, as in the case of nonexpansive mappings with respect to asymptotic regularity, an admissible perturbation of a nonlinear operator enriches this operator. This explains why the admissible perturbations of the demicontractive operators utilized in [2] are enhancing the quality of the edge detection results in medical image processing when using ant-based algorithms.

According to the theoretical results presented above, this fact is mainly due to a kind of mitigation of the iterations for the perturbed test functions, as clearly illustrated by the numerical results presented in the next section.

\section{Admissible Perturbations of Demicontractive Mappings as Test Functions}

In two previous works [2,22], the second author and her collaborators studied some admissible perturbations of demicontractive operators which were used in an ant colony optimization algorithm for edge detection of medical images.

The functions we used in our tests are:

- $\quad T_{\frac{1}{15}}=\left(1-\frac{1}{15}\right) x+\frac{1}{15} T x$, where $T$ is defined in Example 1;

- $\quad T_{\chi}=(1-\chi(x, T x)) x+\chi(x, T x) T x$, where $\chi:[0,1] \rightarrow[0,1]$,

$$
\chi(x, T x)=\frac{x^{2}(T x)^{2}}{\left(1+x^{2}\right)\left(1+(T x)^{2}\right)^{\prime}}
$$

- $\quad T x=\alpha x^{2}$, where $\alpha$ is a parameter which adjusts the shape of the operator, see Example 2 for the case $\alpha=1$;

- $T x=\sin \left(\frac{\pi x}{2 \alpha}\right)$, where $\alpha$ adjusts the shape of the operator.

The demicontractive operator presented in Example 1 is referred to as $T$ starting from this point. To approximate the fixed points, we use Picard iteration and Krasnoselskij 
iteration obtained by applying the admissible perturbation for $\lambda=0.1, \lambda=0.2, \lambda=0.5$ and $\lambda=0.9$, respectively. The Krasnoselskij admissible perturbation operators are denoted as usually by $T_{\lambda}$, with the value of $\lambda$ as index, i.e., $T_{0.1}$ is the admissible perturbation of $T$ for $\lambda=0.1$, and so on.

We performed tests for various values of the initial approximation $x_{0}$, but we present here only the results obtained for $x_{0}=0.6$.

We computed the successive approximation values, $x_{n}$, for $n=\overline{1,60}$ of $T, T_{0.1}, T_{0.2}$, $T_{0.5}$ and $T_{0.9}$. A summary of the results are presented in Table 1 . After 60 iterations, the difference between two successive values was at least $10^{-3}$, i.e., $\left|x_{n+1}-x_{n}\right| \leq 10^{-3}$, for all functions we considered.

Table 1. Values of $x_{n}$ computed using the functions $T, T_{0.9}, T_{0.5}, T_{0.2}$ and $T_{0.1}$ with the initial guess $x_{0}=0.6$.

\begin{tabular}{cccccc}
\hline $\boldsymbol{n}$ & $\boldsymbol{T}$ & $\boldsymbol{T}_{\mathbf{0 . 9}}$ & $\boldsymbol{T}_{\mathbf{0 . 5}}$ & $\boldsymbol{T}_{\mathbf{0 . 2}}$ & $\boldsymbol{T}_{\mathbf{0 . 1}}$ \\
\hline 1 & 0.3982 & 0.4183 & 0.4991 & 0.5596 & 0.5798 \\
\hline 10 & 0.0032 & 0.0062 & 0.0621 & 0.2579 & 0.3970 \\
\hline 20 & $-7.78 \times 10^{-10}$ & $1.81 \times 10^{-8}$ & $1.72 \times 10^{-5}$ & 0.0247 & 0.1331 \\
\hline 30 & $-4.34 \times 10^{-13}$ & $1.31 \times 10^{-11}$ & $1.63 \times 10^{-7}$ & 0.0058 & 0.0671 \\
\hline 40 & $2.25 \times 10^{-19}$ & $2.14 \times 10^{-16}$ & $3.33 \times 10^{-11}$ & $5.82 \times 10^{-4}$ & 0.0231 \\
\hline 50 & $-1.09 \times 10^{-24}$ & $-3.85 \times 10^{-21}$ & $1.29 \times 10^{-14}$ & $6.16 \times 10^{-5}$ & 0.0081 \\
\hline 60 & $2.41 \times 10^{-31}$ & $-3.51 \times 10^{-27}$ & $6.80 \times 10^{-18}$ & $6.66 \times 10^{-6}$ & 0.0029 \\
\hline
\end{tabular}

Figure 1 presents the graphs for the values of $x_{n}$ where $n$ takes successively values from 0 to 60 for the functions $T, T_{0.9}, T_{0.5}, T_{0.2}$ and $T_{0.1}$, respectively.

Figure 2 presents the first 60 values of $x_{n}$ calculated for $T, T_{0.5}, T_{0.2}$ and $T_{0.1}$, respectively. The functions $T_{0.2}$ and $T_{0.9}$ are not presented in Figure 2 due to the fact that their graphs are too close to the graphs of $T_{0.1}$ and $T$, respectively, and so the image would not be clear enough.

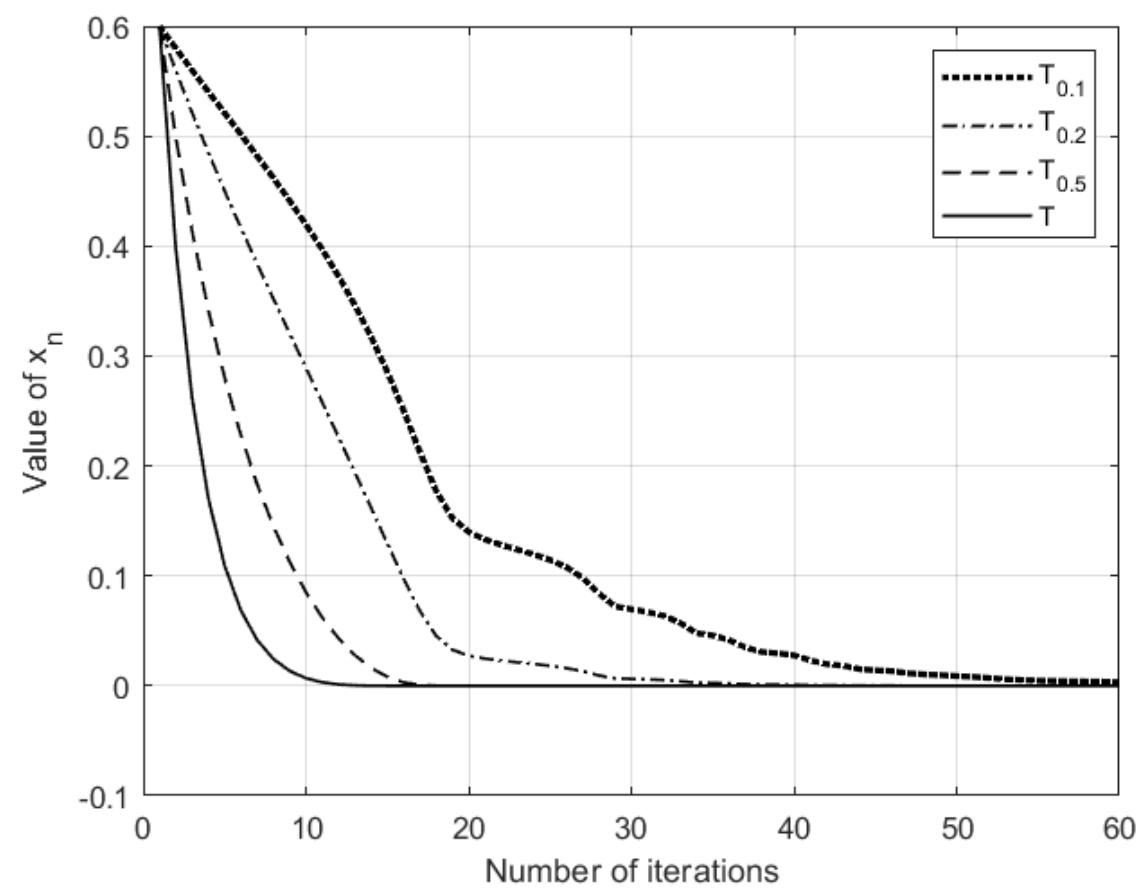

Figure 1. Graph for the successive approximations points corresponding to $T, T_{0.1}, T_{0.2}$ and $T_{0.5}$. 


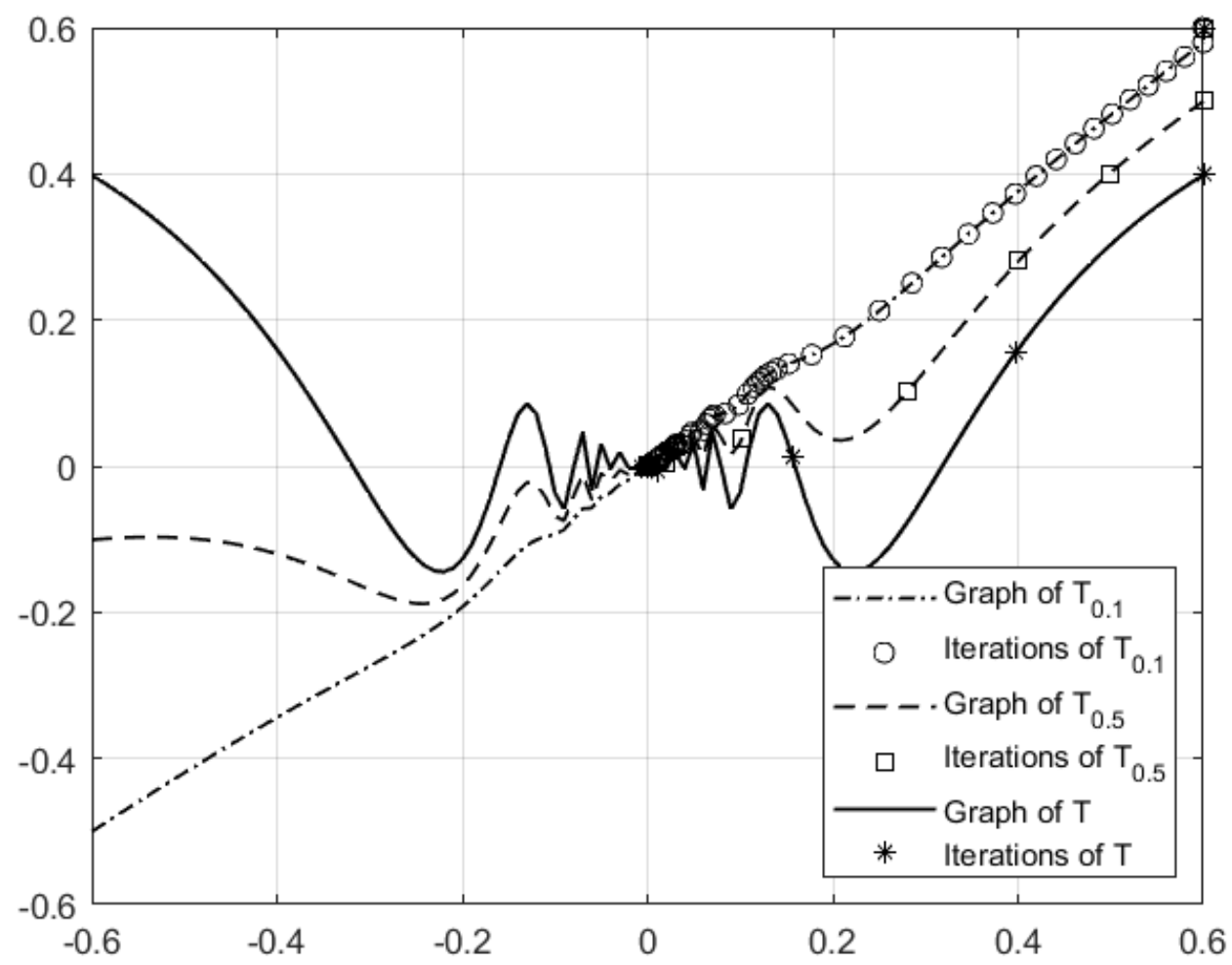

Figure 2. Distribution of iterations $x_{n}$ with respect to the corresponding graphs of operators $T, T_{0.5}$ and $T_{0.1}$.

We note that the convergence of the iterative process associated to the operator $T_{\frac{1}{15}}$ is slower than the one corresponding to the operator $T_{0.1}$. The value of $x_{n}$ generated by $T_{\frac{1}{15}}$ reaches to the fixed point with an error of $10^{-3}$ at the 77th iteration and $x_{n}=0.009$. Obviously, its graph is very similar to the graph of $T_{0.1}$.

In [22], the authors presented facts which indicate that the best results may be obtained when the test functions are admissible perturbations of demicontractive functions and not the function $T$ itself.

When the test function is considered $S$ in Example 2, then the obtained edges were unclear. This is mainly because $S$ is not demicontractive.

We present numerical results produced by the edge detection algorithm described in [2] for a set of medical images using $T, T_{\frac{1}{15}}, T_{0.1}, T_{0.2}, T_{0.5}, T_{0.9}$ and $S$, respectively, as test functions. Various images of the detected edges obtained for the test images using operators $T, T_{\frac{1}{15}}, T_{0.5}$ and $T_{0.9}$ are also presented.

The images used are shown in Figure 3. The images are: Head CT from [23] with a resolution of $128 \times 128$ pixels, an image which is available online for free; Brain CT with a resolution of $128 \times 128$ from personal library; and Hand X-ray from [24] with a resolution of $225 \times 225$, available online for free. The resolution of each image does not influence the conclusions presented, since comparisons are made separately for each image, among results obtained with different test functions.

The images in Figure 3 were used in the edge detection algorithm first presented in [25] and then studied in [2,22].

The present study follows the effect of changing the value of $\lambda$, the variable implied in the admissible perturbation of the demicontractive mapping $T$. 

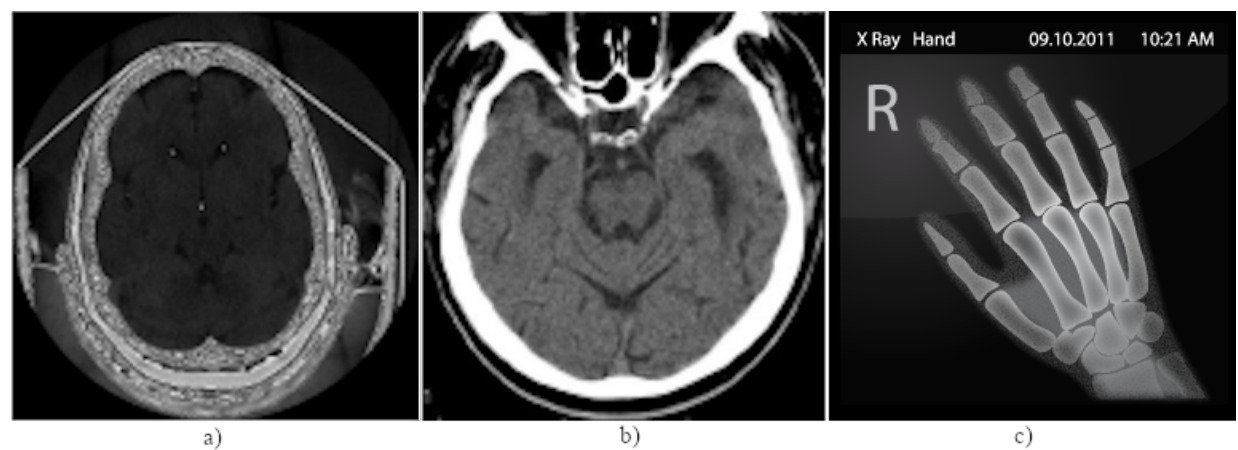

Figure 3. Test-data: medical images (Head_CT (a) [23], Brain_CT (b) and Hand_X-ray [24] (c)).

Each image is represented as a matrix. For each image presented in Figure 3, the values in the matrices are integers with values which range from 0 to 255 inclusively. The values in the matrices corresponding to the generated edges are only values of 0 or 255 exclusively. For the edge, we have the value 0 in the matrix; for the white region, the matrix corresponding value is 255 . We consider the best detected edge the one with the matrix which contains the most zeros. The most similar and the least similar detected edges are also established.

Detected edges for Head CT, Brain CT and Hand X-ray are presented in Figures 4-6, respectively.

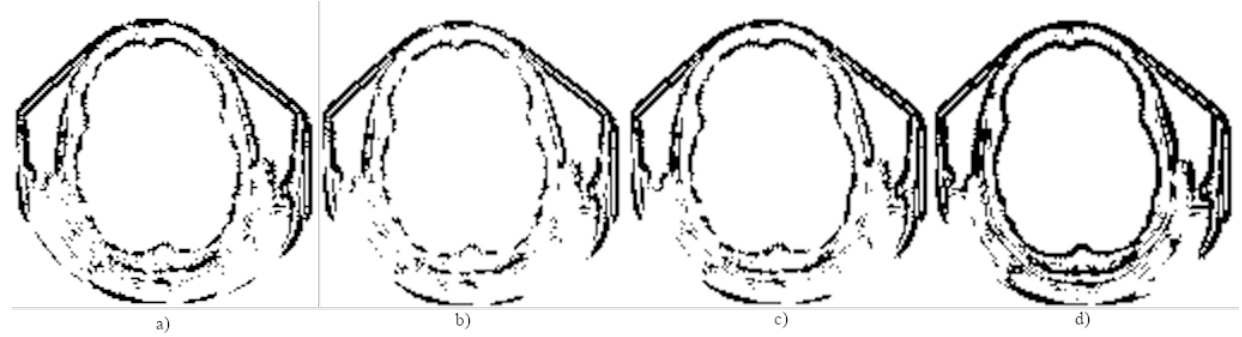

Figure 4. Detected edges for Head CT with test function: (a) $T$; (b) $T_{0.9}$; (c) $T_{0.5}$; and (d) $T_{\frac{1}{15}}$.

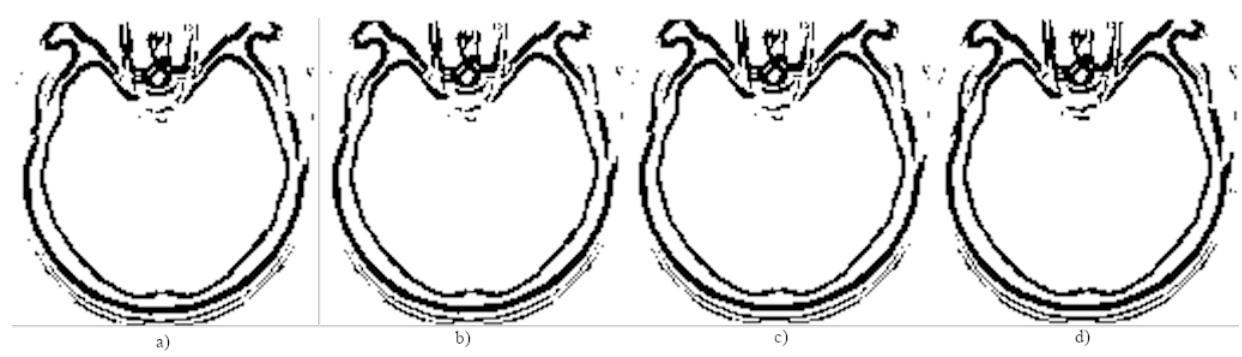

Figure 5. Detected edges for Brain CT with test function: (a) $T$; (b) $T_{0.9} ;$ (c) $T_{0.5}$; and (d) $T_{\frac{1}{15}}$.

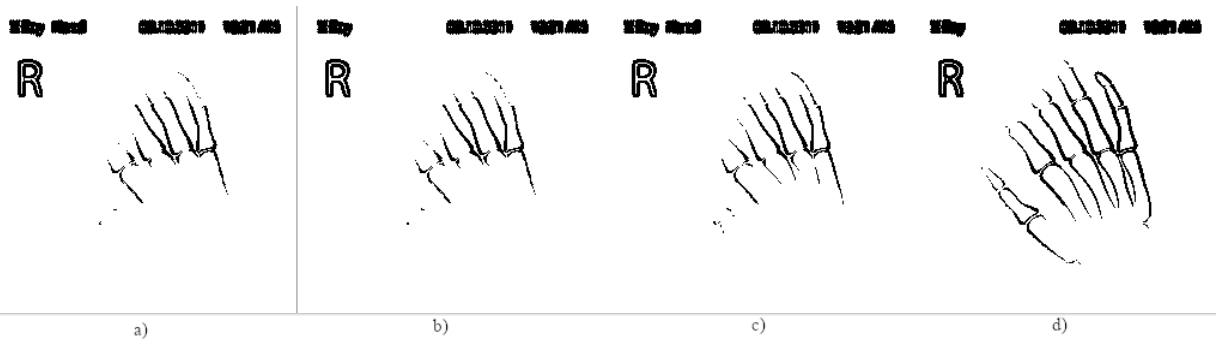

Figure 6. Detected edges for Hand X-ray with test function: (a) $T$; (b) $T_{0.9}$; (c) $T_{0.5}$; and (d) $T_{\frac{1}{15}}$.

Some notations were used: $D$ is the edge matrix generated by operator $T, K_{0.9}$ is the edge matrix generated by operator $T_{0.9}$ and similarly for $K_{0.5}, K_{0.2}, K_{0.1}$ and $K_{\frac{1}{15}}$, respectively, and $N$ is the edge matrix generated by the operator $S$. 
We counted the pixels which form the edge. The results of the counting are presented in Table 2.

Table 2. Number of pixels detected on the edge of Head CT [23], Brain CT and Hand X-ray [24] images.

\begin{tabular}{cccc}
\hline Number of Pixels on the Edge & Head CT & Brain CT & Hand X-ray \\
\hline$D$ & 2233 & 2745 & 3060 \\
\hline$K_{0.9}$ & 1902 & 2737 & 2728 \\
\hline$K_{0.5}$ & 2262 & 2780 & 3172 \\
\hline$K_{0.2}$ & 2879 & 2886 & 3987 \\
\hline$K_{0.1}$ & 3170 & 2940 & 4408 \\
\hline$K_{1}$ & $\mathbf{3 2 3 1}$ & $\mathbf{2 9 6 8}$ & 4605 \\
\hline$N$ & 1969 & 1593 & 1800 \\
\hline
\end{tabular}

It can be easily observed in Figures 4 and 6 that the most compact edge is generated by operator $T_{\frac{1}{15}}$.

In Figure 5, on the other hand, the differences among detected edges is less visible, but we can see in Table 2 that operator $T_{\frac{1}{15}}$ extracted the largest number of pixels and therefore generated the most compact edge.

In addition, in Table 2, the greater numbers of pixels correctly identified are in the row of operator $T_{\frac{1}{15}}$ for all images: Head CT, Brain CT and Hand X-ray. When we consider only the admissible perturbation operators, the extracted edges contain more pixels as the parameter $\lambda$ decreases. Hence, the weakest edge is obtained by $T_{0.9}$, while the most compact one was obtained for $T_{\frac{1}{15}}$.

We should also note that, when we consider the unperturbed functions, namely $T$ and $S$, we observe that the demicontractive function extracted a better edge than $S$, because $S$ is not demicontractive.

Table 3 presents the values obtained by applying the norm (Euclidean norm) to the difference of the edge matrices as the first column of the table indicates. The larger the value is, the less similar the edge contained in the matrix is, and, similarly, the smaller the value is, the more similar the edge is.

For each image, we mark the least and most similar matrices.

The method of detecting edges using ant colonies is, in our opinion, comparable with other methods such as Prewitt, Sobel and Roberts edge detection. To support this statement, we present Figure 7. The edges presented in Figure 7a-c were obtained using in MATLAB the command edge for the edge extraction methods Prewitt, Sobel and Roberts, while Figure $7 \mathrm{~d}$ was obtained by ACO with test function $T_{\frac{1}{15}}$.

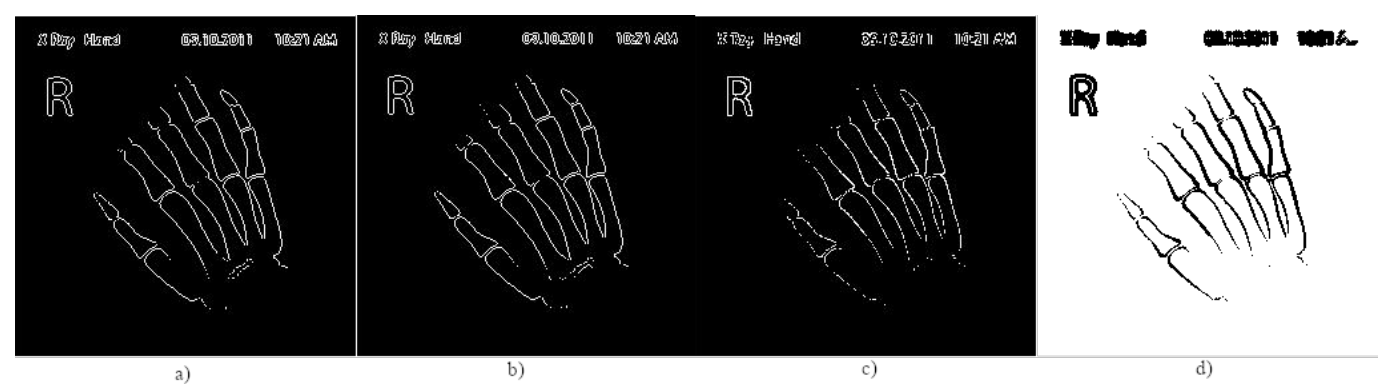

Figure 7. Extracted edges for Hand X-Ray with different edge extraction methods: (a) Prewitt; (b) Sobel; (c) Roberts; and (d) ACO with test function $T_{\frac{1}{15}}$. 
Table 3. Similarity comparison of correctly identified edges for medical images Head CT [23], Brain CT and Hand X-ray [24].

\begin{tabular}{cccc}
\hline Operator/Image & Head CT & Brain CT & Hand X-ray \\
\hline$\left\|D-K_{1}\right\|$ & 2854 & 1054 & 3437 \\
\hline$\left\|D-K_{0.1}\right\|$ & 2710 & 966 & 4388 \\
\hline$\left\|D-K_{0.2}\right\|$ & 2090 & 885 & 6946 \\
\hline$\left\|D-K_{0.5}\right\|$ & 1127 & 671 & 982 \\
\hline$\left\|D-K_{0.9}\right\|$ & 1117 & 413 & 4301 \\
\hline$\|D-N\|$ & 1164 & 2987 & 4481 \\
\hline$\left\|K_{\frac{1}{15}}-K_{0.1}\right\|$ & 534 & 471 & 4826 \\
\hline$\left\|K_{\frac{1}{15}}-K_{0.2}\right\|$ & 1271 & 610 & 6803 \\
\hline$\| K_{\frac{1}{15}}-K_{0.5}$ & 2799 & 921 & 3048 \\
\hline$\| K_{\frac{1}{15}}-K_{0.9}$ & 3558 & 1022 & 4872 \\
\hline$\left\|K_{1}-N\right\|$ & 3412 & 3541 & 5274 \\
\hline$\left\|K_{0.1}-K_{0.2}\right\|$ & 1113 & 529 & 5533 \\
\hline$\left\|K_{0.1}-K_{0.5}\right\|$ & 2641 & 805 & 4335 \\
\hline$\left\|K_{0.1}-K_{0.9}\right\|$ & 3399 & 936 & 3429 \\
\hline$\left\|K_{0.1}-N\right\|$ & 3250 & 3473 & 4936 \\
\hline$\left\|K_{0.2}-K_{0.5}\right\|$ & 1951 & 715 & 6841 \\
\hline$\left\|K_{0.2}-K_{0.9}\right\|$ & 2714 & 823 & 5438 \\
\hline$\left\|K_{0.2}-N\right\|$ & 2538 & 3367 & 5299 \\
\hline$\left\|K_{0.5}-K_{0.9}\right\|$ & 1261 & 615 & 43948 \\
\hline$\left\|K_{0.5}-N\right\|$ & 1022 & 2979 & \\
\hline$\left\|K_{0.9}-N\right\|$ & 898 & & \\
\hline
\end{tabular}

Of course, improvements can be made in order to obtain better edges. Such improvements may be:

- applying filters to reduce the noise in the source image;

- segmenting the image before edge extraction, extracting the edge and reunite the obtained edges for more details;

- finding a method to eliminate the possible noise in the edge.

In our future research, we intend to use the presented edge detection method in Canny edge detection at the step in which intensity gradient of the image is found.

Edge detection methods are still a domain in which progress is desirable. The aim is to improve the quality in such a way that a view of the generated edges should offer a better understanding of the original image.

\section{Conclusions}

(1) We show, both analytically and empirically, that one can improve ant-based algorithms for image edge detection by using admissible perturbations of demicontractive mappings as test functions. For another possible way to find more selective test functions that can improve ant-based algorithms for image edge detection, we refer to the classes of 
the so-called enriched mappings—which are in fact admissible perturbations of various contractive operators - which were very recently studied by the authors of [26-34].

(2) Thus, we support theoretically the empirical results reported in the recent papers [2,22], and we also performed relevant new numerical experiments and comparison studies of the numerical results obtained. The edge detection process reported in our study considers both symmetric (Head CT and Brain CT) and asymmetric (Hand X-ray) medical images.

(3) Our extensive numerical experiments clearly indicate that the ant-based algorithms are significantly improved by using as test functions various admissible perturbations of demicontractive mappings and also that a less accurate edge is extracted when using non-demicontractive test functions.

(4) We also raise an interesting open question for further studies. As observed from the experimental results obtained, by considering two different test functions, the extracted edge by the first function could be essentially different from the edge extracted by the second function. Thus, even if the edge extraction rate of a certain function is not very high, it will be useful to find a way to combine it with another (better) test function to form a single one test function with an even better edge extraction rate.

(5) It will also be of real theoretical and practical interest to find some of the analytical reasons for the above property.

Author Contributions: Both authors contributed equally to this work. Both authors have read and agreed to the published version of the manuscript.

Funding: The authors were financially supported by Department of Mathematics, Faculty of Sciences, North University Centre at Baia Mare, Technical University of Cluj-Napoca.

Data Availability Statement: Data supporting reported results which are not public can be obtained at request from the second author.

Acknowledgments: This research was supported by the Department of Mathematics and Computer Science, Technical University of Cluj-Napoca, North University Centre at Baia Mare, through the Internal Research Grant No. 5/2021. The authors are extremely grateful to both referees for the pertinent and constructive comments and suggestions which contributed to the improvement of the manuscript.

Conflicts of Interest: The authors declare no conflict of interest.

\section{References}

1. Byrne, C.L. Applied Iterative Methods; A K Peters, Ltd.: Wellesley, MA, USA, 2008.

2. Ţicală, C.; Zelina, I.; Pintea, C.-M. Admissible perturbation of demicontractive operators within ant algorithms for medical images edge detection. Mathematics 2020, 8, 1040. [CrossRef]

3. Măruşter, Ş. Sur le calcul des zéros d'un opérateur discontinu par itération. Canad. Math. Bull. 1973, 16, 541-544. [CrossRef]

4. Hicks, T.L.; Kubicek, J.D. On the Mann iteration process in a Hilbert space. J. Math. Anal. Appl. 1977, 59, 498-504. [CrossRef]

5. Maingé, P.-E. A hybrid extragradient-viscosity method for monotone operators and fixed point problems. SIAM J. Control Optim. 2008, 47, 1499-1515. [CrossRef]

6. Măruşter, Ş. The solution by iteration of nonlinear equations in Hilbert spaces. Proc. Am. Math. Soc. 1977, 63, 69-73. [CrossRef]

7. Qin, L.-J.; Wang, G. Multiple-set split feasibility problems for a finite family of demicontractive mappings in Hilbert spaces. Math. Inequal. Appl. 2013, 16, 1151-1157. [CrossRef]

8. Suantai, S.; Phuengrattana, W. A hybrid shrinking projection method for common fixed points of a finite family of demicontractive mappings with variational inequality problems. Banach J. Math. Anal. 2017, 11, 661-675. [CrossRef]

9. Thong, D.V.; Hieu, D.V. Modified subgradient extragradient algorithms for variational inequality problems and fixed point problems. Optimization 2018, 67, 83-102. [CrossRef]

10. Vuong, P.T.; Strodiot, J.J.; Nguyen, V.H. On extragradient-viscosity methods for solving equilibrium and fixed point problems in a Hilbert space. Optimization 2015, 64, 429-451. [CrossRef]

11. Browder, F.E.; Petryshyn, W.V. The solution by iteration of nonlinear functional equations in Banach spaces. Bull. Am. Math. Soc. 1966, 72, 571-575. [CrossRef]

12. Krasnosel'skiǐ, M.A. Two remarks about the method of successive approximations. Uspehi Mat. Nauk 1955, 10, 123-127.

13. Rus, I.A. An abstract point of view on iterative approximation of fixed points: Impact on the theory of fixed point equations. Fixed Point Theory 2012, 13, 179-192. 
14. Berinde, V. Convergence theorems for fixed point iterative methods defined as admissible perturbations of a nonlinear operator. Carpathian J. Math. 2013, 29, 9-18. [CrossRef]

15. Berinde, V.; Khan, A.R.; Păcurar, M. Convergence theorems for admissible perturbations of $\phi$-pseudocontractive operators. Miskolc Math. Notes 2014, 15, 27-37. [CrossRef]

16. Berinde, V.; Măruşter, S..; Rus, I.A. An abstract point of view on iterative approximation of fixed points of nonself operators. J. Nonlinear Convex Anal. 2014, 15, 851-865.

17. Toscano, E.; Vetro, C. Admissible perturbations of $\alpha-\psi$-pseudocontractive operators: convergence theorems. Math. Methods Appl. Sci. 2017, 40, 1438-1447. [CrossRef]

18. Toscano, E.; Vetro, C. Fixed point iterative schemes for variational inequality problems. J. Convex Anal. 2018, $25,701-715$.

19. Ticală, C. Approximating fixed points of demicontractive mappings by iterative methods defined as admissible perturbations. Creat. Math. Inform. 2016, 25, 121-126.

20. Ticală, C. Approximating fixed points of asymptotically demicontractive mappings by iterative schemes defined as admissible perturbations. Carpathian J. Math. 2017, 33, 381-388. [CrossRef]

21. Petruşel, A.; Rus, I.A. An abstract point of view on iterative approximation schemes of fixed points for multivalued operators. $J$. Nonlinear Sci. Appl. 2013, 6, 97-107. [CrossRef]

22. Țicală, C.; Zelina, I. New ant colony optimization algorithm in medical images edge detection. Creat. Math. Inform. 2020, 29, 101-108. [CrossRef]

23. Head CT. Online Medical Free Image. Available online: http://www.libpng.org/pub/png/pngvrml/ct2.9-128x128.png (accessed on 1 May 2020).

24. X-ray Hand. Vista Medical Pack. License: Free for Non Commercial Use. id, 236487. Available online: https://www.iconspedia. $\mathrm{com} /$ (accessed on 1 May 2020).

25. Tian, J.; Yu,W.; Xie, S. An ant colony optimization algorithm for image edge detection. In Proceedings of the IEEE Congress on Evolutionary Computation (IEEEWorld Congress on Computational Intelligence), Hong Kong, China, 1-6 June 2008; pp. 751-756.

26. Berinde, V. Weak and strong convergence theorems for the Krasnoselskij iterative algorithm in the class of enriched strictly pseudocontractive operators. An. Univ. Vest Timiş. Ser. Mat.-Inform. 2018, 56, 13-27. [CrossRef]

27. Berinde, V. Approximating fixed points of enriched nonexpansive mappings by Krasnoselskij iteration in Hilbert spaces. Carpathian J. Math. 2019, 35, 293-304. [CrossRef]

28. Berinde, V. Approximating fixed points of enriched nonexpansive mappings in Banach spaces by using a retraction-displacement condition. Carpathian J. Math. 2020, 36, 27-34. [CrossRef]

29. Berinde, V.; Păcurar, M. Approximating fixed points of enriched contractions in Banach spaces. J. Fixed Point Theory Appl. 2020, 22, 1-10. [CrossRef]

30. Berinde, V.; Păcurar, M. Kannan's fixed point approximation for solving split feasibility and variational inequality problems. J. Comput. Appl. Math. 2021, 386, 113217. [CrossRef]

31. Berinde, V.; Păcurar, M. Fixed point theorems for Chatterjea type mappings in Banach spaces. J. Fixed Point Theory Appl. 2021, under review.

32. Berinde, V.; Păcurar, M. Krasnoselskij-type algorithms for variational inequality problems and fixed point problems in Banach spaces. arXiv 2021, arXiv:2103.10289.

33. Berinde, V.; Păcurar, M. Existence and Approximation of Fixed Points of Enriched Contractions and Enriched $\varphi$-Contractions. Symmetry 2021, 13, 498. [CrossRef]

34. Berinde, V.; Păcurar, M. Fixed Points Theorems for Unsaturated and Saturated Classes of Contractive Mappings in Banach Spaces. Symmetry 2021, 13, 713. [CrossRef] 